\title{
La mucoviscidose au fil du temps
}

Prof. Nicolas Regamey, Lucerne; Dr Anne Mornand, Genève; Prof. Jürg Barben, Saint-Gall

L'exemple de la maladie génétique la plus fréquente dans la population caucasienne - la mucoviscidose ou fibrose kystique - illustre parfaitement ce que la Suisse reçoit en retour pour les dépenses croissantes de santé. Au cours des 30 dernières années, les progrès médicaux ont considérablement amélioré l'espérance et la qualité de vie des personnes atteintes de mucoviscidose, avec des effets positifs non seulement pour les patients concernés, mais aussi pour l'ensemble de la société.

La mucoviscidose, également appelée fibrose kystique, est l'une des maladies génétiques les plus fréquentes, qui reste malheureusement incurable aujourd'hui, même si les possibilités de traitement ne cessent de s'améliorer. Environ 5\% de la population est porteuse le plus souvent sans le savoir - d'une mutation d'un chromosome, mais ne développe pas la maladie car le deuxième chromosome est intact. Chez un nouveauné sur 3000 environ, les deux parents transmettent chacun un chromosome modifié, et l'enfant est atteint de mucoviscidose. Un millier de personnes concernées par cette maladie, qui touche aujourd'hui déjà plus d'adultes que d'enfants, vivent actuellement en Suisse. Chez ces personnes, à cause de l'anomalie génétique, les cellules produisent des sécrétions trop visqueuses qui entravent en premier lieu le fonctionnement pulmonaire et digestif. En règle générale, la mucoviscidose se manifeste dès la première année par une toux et un encombrement, des douleurs abdominales ainsi qu'une mauvaise prise de poids chez les nouveau-nés. Dans les années qui suivent, ces personnes souffrent surtout d'inflammations et de lésions du tissu pulmonaire ainsi que d'une insuffisance pancréatique entraînant une prévalence du diabète.

\section{L'espérance de vie des personnes atteintes de mucoviscidose a plus que doublé}

Alors que l'espérance de vie des personnes atteintes de mucoviscidose était d'à peine 20 ans dans les années 80 , elle est aujourd'hui de presque 50 ans et ne cesse de croître. Il y a 30 ans, les pneumologues pédiatriques devaient annoncer aux parents d'un enfant frappé par cette maladie qu'il mourrait probablement avant d'atteindre l'âge adulte. Pour ces pédiatres, il était alors fréquent d'accompagner ces petits patients dans la dernière phase de leur vie et de les voir mourir de la maladie. Heureusement, ce n'est aujourd'hui que rare- ment le cas puisque la plupart des personnes atteintes de mucoviscidose atteignent l'âge adulte et beaucoup d'entre elles avec une fonction pulmonaire presque normale.

\section{La qualité de vie des patients et de leurs proches s'est aussi considérablement améliorée}

En plus d'une durée de vie allongée, les personnes atteintes de mucoviscidose jouissent aujourd'hui d'une bien meilleure santé qui leur permet souvent de participer pleinement à la vie sociale et professionnelle. Ces personnes travaillent actuellement dans presque toutes les branches professionnelles, font du sport et

Un millier de personnes concernées par cette maladie, qui touche aujourd'hui déjà plus d'adultes que d'enfants, vivent actuellement en Suisse.

fondent souvent une famille. Elles redonnent ainsi à la société une part de ce qu'elles ont reçu sur les plans humain, social et financier.

La qualité de vie améliorée de ces personnes est due non seulement à un meilleur traitement des symptômes, mais aussi à une amélioration des techniques de soins. Les traitements ambulatoires rendus aujourd'hui possibles par la médecine moderne permettent le plus souvent d'éviter aux enfants des hospitalisations éprouvantes pour eux-mêmes et leurs familles. Dorénavant, les traitements antibiotiques intraveineux n'impliquent plus systématiquement deux semaines d'hospitalisation, mais peuvent se faire à domicile. Les enfants et les jeunes adultes sont par conséquent moins absents de l'école ou du travail. Par ailleurs, certaines thérapies ont pu être simplifiées, à l'instar des traitements par inhalation qui doivent être 
effectués plusieurs fois par jour. Sur l'ensemble d'une journée ou des années, cela fait une grande différence si une nébulisation médicamenteuse qui durait $15 \mathrm{mi}-$ nutes il y a 20 ans peut aujourd'hui être réalisée en 5 minutes. De nombreux médicaments pour inhalation sont aujourd'hui aussi disponibles sous forme de poudre, ce qui simplifie et raccourcit leur prise.

\section{Les raisons d'une espérance et d'une qualité de vie améliorées}

Une connaissance de plus en plus approfondie de la maladie constitue un des facteurs principaux d'amélioration de sa prise en charge. La compréhension des mécanismes physiopathologiques permet désormais de façon précoce le dépistage de la maladie et le traitement des complications. Par exemple, le diabète associé à une mucoviscidose est recherché très tôt, ce qui permet la mise en place rapide d'un traitement adapté. Des médicaments inhalés comme la solution saline hypertonique ou la DNAse recombinante peuvent également être utilisés à titre préventif pour ralentir l'atteinte pulmonaire.

En 1989, le défaut génétique à l'origine de la maladie a pu être identifié (anomalie du gène CFTR qui code pour un canal régulant le transport du chlore à travers la membrane des cellules). Des thérapies ont donc pu être développées non seulement pour pallier les conséquences relatives au défaut de transport du chlore,

\section{Ces personnes travaillent actuellement dans presque toutes les branches professionnelles, font du sport et fondent souvent une famille.}

mais aussi pour tenter de corriger ce défaut de transport à la racine. Des médicaments récents, appelés modulateurs et correcteurs de CFTR, sont susceptibles d'améliorer la fonction du canal CFTR, et donc d'influer ou d'atténuer de manière déterminante l'évolution de la maladie, du moins pour certaines mutations. Hormis le développement de ces nouveaux médicaments, les personnes atteintes de mucoviscidose bénéficient également des progrès réalisés dans la médecine de transplantation. Une transplantation du poumon peut en effet prolonger considérablement la vie de ces patients tout en améliorant leur qualité de vie.
Grâce au programme de dépistage national introduit en 2011, il est aujourd'hui possible de diagnostiquer la mucoviscidose chez les nouveau-nés et d'épargner ainsi aux familles un chemin souvent long et laborieux, mais aussi onéreux, menant au diagnostic; cette mesure permet également un traitement précoce de la maladie et un meilleur pronostic.

Enfin, notons que la mise en place d'une prise en charge pluridisciplinaire a elle aussi permis une amélioration considérable des soins. Une équipe composée de pédiatres, de pneumologues, d'infirmiers, de physiothérapeutes, d'assistants sociaux, de psychologues et de nutritionnistes collabore étroitement avec des représentants d'autres disciplines pour une prise en charge complète et globale des personnes atteintes afin d'améliorer leur espérance et leur qualité de vie. Les coûts occasionnés sont donc à mettre en parallèle avec une durée de vie nettement plus longue et améliorée de ces patients, ce qui représente non seulement un gain pour les patients et leurs proches, mais aussi pour la société dans son ensemble.

\section{Résumé - Bénéfices}

Au cours des 20 dernières années, les progrès médicaux évoqués plus haut ont tellement amélioré le pronostic des personnes atteintes de mucoviscidose que la maladie, autrefois exclusivement pédiatrique, est devenue une maladie de l'adulte. Autrefois, la maladie frappait les enfants, qui nécessitaient une prise en charge et des soins $24 \mathrm{~h}$ sur 24 , et qui mouraient souvent avant d'avoir atteint l'âge adulte; aujourd'hui en Suisse, plus de la moitié des personnes concernées par cette maladie sont des adultes bénéficiant d'une qualité de vie nettement améliorée. Il est désormais également possible de procéder à une transplantation d'organe susceptible de prolonger la vie de plusieurs années avec une bonne qualité de vie. Les adultes atteints de mucoviscidose peuvent, en règle générale, exercer un métier et participer à la vie sociale. Ces personnes, leurs familles et leur entourage sont très reconnaissants de ces avancées médicales qui, certes, coûtent cher à la société, mais permettent aussi un retour non négligeable! 